\title{
PSIDIUM GUAJAVA L. EXTRACT INCREASES PLATELET COUNT THROUGH ENHANCEMENT OF STEM CELL FACTOR EXPRESSION IN THROMBOCYTOPENIC MICE MODEL
}

\author{
NUR ATIK ${ }^{*}$, SUSAN TARAWIFA ${ }^{2}$, ERDA AVRIYANTI ${ }^{3}$, ANDRI REZA RAHMADI ${ }^{4}$, DANY HILMANTO ${ }^{5}$ \\ 1Departement of Anatomy, Physiology and Cellular Biology, Faculty of Medicine, Universitas Padjadjaran, Bandung,

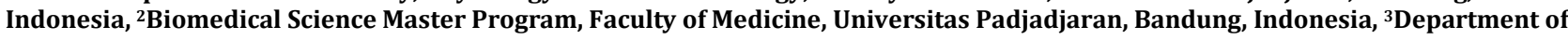 \\ Dermatology and Venereology, Faculty of Medicine, Universitas Padjadjaran/Dr. Hasan Sadikin General Hospital, Bandung, \\ Indonesia, ${ }^{4}$ Department of Internal Medicine, Faculty of Medicine, Universitas Padjadjaran/Dr. Hasan Sadikin General Hospital, Bandung, \\ Indonesia, ${ }^{5}$ Department of Pediatrics, Faculty of Medicine, Universitas Padjadjaran/Dr. HasanSadikin General Hospital, Bandung, \\ Indonesia \\ Email: n.atik@unpad.ac.id
}

Received: 13 Jul 2017 Revised and Accepted: 22 Nov 2017

\section{ABSTRACT}

Objective: The purpose of this study was to determine the mechanism through which guava extract increases platelet count.

Methods: Twenty male Swiss webster mice were divided into 4 group. Group I received $2.8 \mathrm{mg} / 20 \mathrm{~g}$ body weight of quinine and $1 \mathrm{ml}$ of distilled water. Group II received $2.8 \mathrm{mg} / 20 \mathrm{grBW}$ of quinine and $0.785 \mathrm{mg} / 20 \mathrm{grBW}$ of guava extract. Group III received $1 \mathrm{ml}$ distilled water and 0.785 $\mathrm{mg} / 20 \mathrm{grBW}$ of guava extract and Group IV only received $1 \mathrm{ml}$ distilled water. After 14 days** treatment, platelet count was measured using RaasEcker method. SCF and TPO mRNA expressions in mice liver were assessed using qRT-PCR.

Results: The data showed that quinine administration reduced platelet count significantly $(\mathrm{P}<0.05)$. Psidium guajava L. extraction significantly enhanced platelet count in mice that were treated with quinine compared with quinine group $(p<0.05)$. The mechanism was analysed further and our RT PCR data showed that stem cell factor mRNA expression significant increased $(\mathrm{p}<0.05)$ in quinine plus guava extract group compared to quinine group.

Conclusion: It indicated guava extract role in increasing megakaryopoiesis which resulted in an increase of platelet count.

Keywords: Guava extract, Platelet, Stem cell factor, Thrombopoietin

(C) 2018 The Authors. Published by Innovare Academic Sciences Pvt Ltd. This is an open-access article under the CC BY license (http://creativecommons.org/licenses/by/4.0/) DOI: http://dx.doi.org/10.22159/ijpps.2018v10i1.21317

\section{INTRODUCTION}

Platelets are small anucleate cell fragments that are discoid in shape, 1 to $3 \mu \mathrm{m}$ in diameter. Formed from the cytoplasm of megakaryocytes (MKs) which reside in the bone marrow, they are indispensable for processes such as haemostasis, wound healing, angiogenesis, inflammation, and innate immunity [1]. The average platelet number in humans ranges from $150 \times 10^{9}$ to $400 \times 10^{9}$ per liter [2]

Platelet generation is a two-stage process involving the differentiation of hematopoietic stem cells into mature megakaryocytes and release of platelets from MKs [3]. Stem cell factor (SCF) and thrombopoietin (TPO) are critical cytokines in regulating stem cell proliferation and survival of progenitor cell during hematopoiesis. In addition, TPO is an important megakaryocytic cytokine affecting the megakaryocytic differentiation [4].

Guava (Psidium guajava L.) is a plant widely used in local traditional medicine. Their fruit and leaves have been studied for their medicinal values such as in the treatment of clinical dengue infection, and for its anti-microbial, antiplasmodial, cytotoxic, antispasmodic, anti-cough, and anti-diabetic effects $[5,6]$. In relation to treating dengue, guava juice and leaves extract has been demonstrated to improve platelet count both in human and animal models $[7,8]$. Additionally, guava extract has been shown to increase the number of megakaryocyte in mice [9]. However, the underlying mechanism has not been investigated.

A possible mechanism may involve the thrombopoiesis cytokines. Quercetin has been reported to increase the level of mRNA expression of SCF in the bone marrow stromal cell in a dosedependent manner [10]. Subsequently, SCF stimulates platelet production by increasing mast cell IL-6 secretion that induces liver to secrete thrombopoietin [11-13]. Thus, it is expected that guava extract may also induce SCF and TPO, resulting in increased platelet number. The aim of this study was to determine the effect of guava extract on mRNA expression of SCF and TPO in the liver of mice.

\section{MATERIALS AND METHODS}

\section{Chemical and reagents}

SV total RNA isolation system kit, reverse transcriptase, and all primers were purchased from Promega, USA. EDTA tubes were purchased from BD, USA. Other chemicals that used for analytical were purchased from Merck, USA.

\section{Collection and extraction of plant materials}

Fresh guava fruits were collected from a farm in Dukuh Waluh village, Purwokerto, Central Java, Indonesia. Guava extract was obtained at the Research Laboratory of the Department of Chemistry, Padjadjaran University, Bandung, Indonesia. Specifically, the fruits were washed and dried using an electric oven at $40-60{ }^{\circ} \mathrm{C}$ for $24 \mathrm{~h}$, pulverized using an electric grinder, and macerated in $96 \%$ ethanol for $24 \mathrm{~h}$. The ethanol extract liquid was evaporated in a rotary evaporator $\left(80^{\circ} \mathrm{C}\right)$. The extract and the fractions were stored at $4{ }^{\circ} \mathrm{C}$ prior to use.

\section{Animals}

Male Swiss webster mice aged 8-12 w, weighing 20-30 g, were used in the study. They were maintained under standard laboratory conditions of $25 \pm 2{ }^{\circ} \mathrm{C}, 55-60 \%$ relative humidity and $12 \mathrm{~h}$ light $/ 12 \mathrm{~h}$ dark cycle. Animals were fed with commercial pellets diet and water ad libitum. The number of mice required for the study was determined using resource equation method. The health of animal subjects was ensured prior to the administration of intervention, as 
measured by the absence of wound, clean hair, and normal physical functioning as inclusion criteria. Mice that became sick or had a more than $10 \%$ body weight reduction after adaptationwere excluded from the experiment. Approval for animal studies was obtained from the ethics committee of Padjadjaran University, Bandung, Indonesia (Number of ethical clearance: 0317010032).

\section{Experimental design}

The mice $(n=20)$ were divided into four groups of five. The first group received $2.8 \mathrm{mg} / 20 \mathrm{~g}$ body weight of quinine orally for $14 \mathrm{~d}$ and $1 \mathrm{ml}$ of distilled water orally for $5 \mathrm{~d}$. The second group received $2.8 \mathrm{mg} / 20 \mathrm{~g}$ body weight of quinine orally for $14 \mathrm{~d}$ and 0.785 $\mathrm{mg} / 20 \mathrm{~g}$ body weight of guava extract orally for $5 \mathrm{~d}$. Group three received $1 \mathrm{ml}$ distilled water orally for $14 \mathrm{~d}$ and $0.785 \mathrm{mg} / 20 \mathrm{~g}$ body weight of $P$. guajava L. extract orally for $5 \mathrm{~d}$. Lastly, group four received $1 \mathrm{ml}$ distilled water orally for $19 \mathrm{~d}$ (fig. 1).

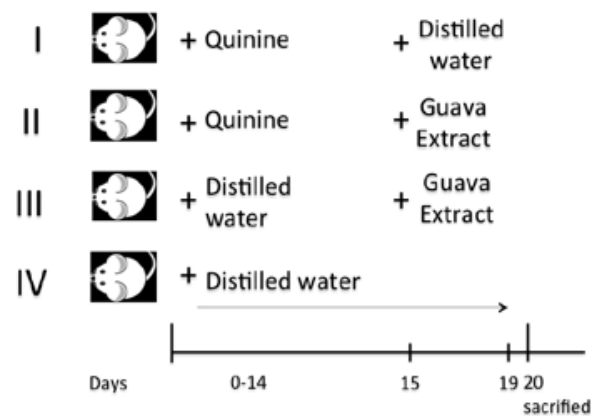

Fig. 1: Experimental design, male mice $(n=20)$ were divided into four groups, two groups received $2.8 \mathrm{mg} / 20 \mathrm{~g}$ body weight of quinine for $14 \mathrm{~d}$ to induce thrombocytopenia and two other groups received distilled water to obtain normal platelet count. In addition, groups II and III received $0.785 / 20 \mathrm{~g}$ body weight of guava extract to measure its effect on platelet count, mRNA SCF and TPO expression. On day, all of the mice were euthanized and mRNA expression was evaluated using qRT-PCR

\section{Sample collection}

At day 20 , the mice were euthanized using a $0.1 \mathrm{ml} / 20 \mathrm{~g}$ body weight combination of ketamine and xylazine administered intraperitoneally. Blood samples were collected by cardiac puncture using sterile needle and syringe in EDTA tubes. Liver tissues were also collected for RNA extraction.

\section{Platelet counting}

Platelet counts were performed using Raas-Ecker method. Following previously described procedures, samples were diluted using a mixture of sodium citrate, $40 \%$ formaldehyde, brilliant cresyl blue, and deionised water. Using clean RBC pipette and Neubauer's chamber, mice fingertip was punctured and blood was collected exactly up to the 0.5 mark on the RBC pipette. Subsequently, the blood sample was rapidly diluted with the Raas-Ecker fluid up to the 101 mark of the pipette and shaken for $1 \mathrm{~min}$. Approximately 3-5 drops of the solution were discarded from the pipette and move into Neubauer's chamber then, cover the chamber. After $15 \mathrm{~min}$, the number of platelets was counted under high power objective in all of the 25 medium squares of the RBC square, that is, in $1 \mathrm{~mm} 3$ area or $1 / 10 \mathrm{~mm}^{3}$ volume. The platelet count was measured using the formula [14]:

Platelet number $/ \mu \mathrm{l}$ or $\mathrm{mm}^{3}$ of blood $=\frac{\text { number of platelets count } \mathrm{x} \text { dilution }}{\text { volume of flud }}$

\section{RNA isolation and quantitative RT-PCR}

Total RNA was extracted from $30 \mathrm{mg}$ of liver tissue using SV total RNA Isolation System Kit according to the manufacturer's instructions. Total RNA was primed with random primers to synthesize first-strand cDNA using reverse transcriptase. The primers used for PCR (5'-3') were as follow [15-16]:

\section{TPO: Forward: TCGAAGCTTGGCCAGAATGGAGCTGACTG}

\section{Reverse: ATAAGATCTGCGCTATGTTTCCTGAGACA}

SCF: Forward: CCTTATGAAGAAGACACAAACTTGG

Reverse: CCATCCCGGCGACATAGTTGAGGG

GAPDH: Forward: CCCATCACCATCTTCCAGGAGC

Reverse: CCAGTGAGCTTCCCGTTCAGC

\section{Statistical analysis}

Statistical analyses were presented as mean \pm SD. The differences between mean value were evaluated using the Student's t-test for unpair data, and $P$ value $<0.05$ was considered statistically significant.

\section{RESULTS}

\section{Fruit identification}

To know the characteristic of $P$. guajava $L$, we collected fruits from a farmland in Dukuh Waluh village, Purwokerto, Central Java. After evaluated we found that fruits have taxonomy as Psidium guajava $L$. with Myrtaceae as family and Myrtalesas order.

\section{Platelets count}

Previous data study showed that guava juice increased platelet number in mice [8]. To evaluate the effect of guava extract in increasing platelet count, we compared the counts from mice after treatment with quinine and guava extracts. We found that quinine administration significantly reduced platelet count when compared with control $(p<0.05)$. On the other hand, guava extract significantly enhanced platelet count in mice that were treated with quinine $(p<0.05)$ while it had no significant effect in mice that received distilled water ( $p>0.05$ ) (fig. 2$)$.

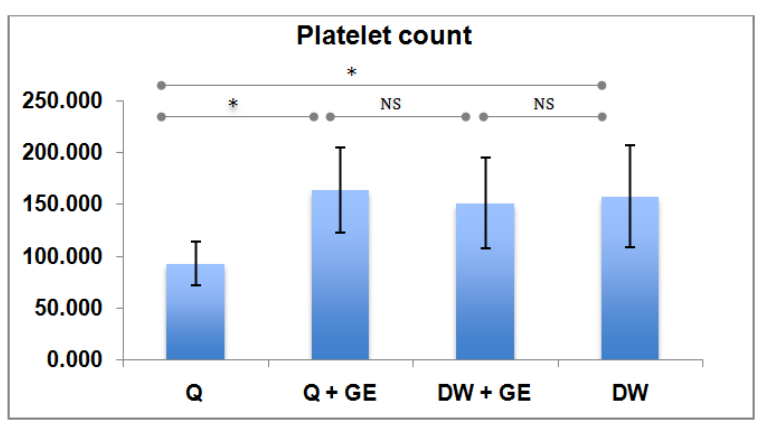

Fig. 2: Guava extract increase platelet count, mice bloods were collected by cardiac puncture, analysis using independent t-test found that guava extract significantly increase platelets count in mice were treated with quinine compared to control

$(p<0.05)$. Data were presented from 5 independent mice in each group. Q: quinine, GE: guava extract, DW: distilled water, "significancy $P<0.05$, NS: not significant

\section{SCF expression}

The increase in a number of platelets increased count may be due to the rise of several molecules such as SCF [4]. To determine the molecular mechanism of platelet production in mice model after treatment with guava extract, we evaluated the expression of SCF mRNA using qRT-PCR. Following oral administration of quinine, in spite of a significant effect on platelet count when compared to the group that received distilled water, statistically significant effect on SCF and TPO mRNA expressions were not observed ( $p>0.05)$. On the contrary, guava extract administration significantly enhanced expression of SCF mRNA in quinine group mice liver $(p<0.05)$ (fig. 3).

\section{TPO expression}

The previous study showed that platelet production was modulated by TPO through the activation of SCF [11-13]. We determined the 
expression of TPO mRNA using Q-PCR and discovered that guava extract had no effect on TPO mRNA expression (fig. 4). However, quantification by Livak method demonstrated that the expression of TPO mRNA was increased by 4.8 fold in mice that received quinine and guava extract compared to that received quinine only

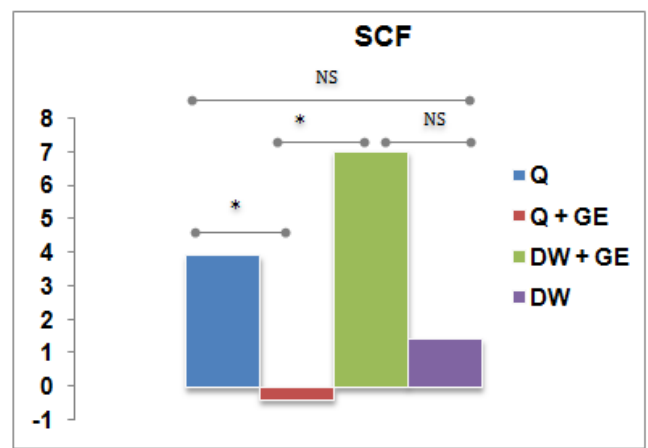

Fig. 3: SCF mRNA expression was enhanced after guava extract administration, expression of SCF mRNA from mice liver was evaluated using quantitative RT-PCR, the result showed that SCF mRNA expression significantly increased in mice that received quinine and guava extract $(p<0.05)$ compared to the mice that received quinine only, Data were presented from 5 independent mice in each group.

Q: quinine, GE: guava extract, DW: distilled water, *significancy $P<0.05$, NS: not significant

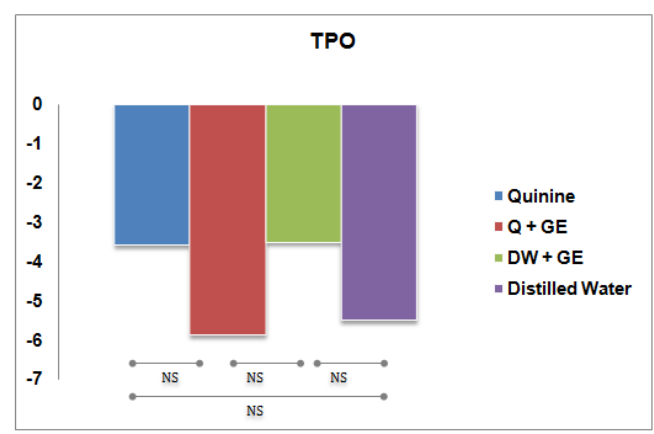

Fig. 4: TPO mRNA expression level, Guava extract had no effect on TPO mRNA expression, as evaluated using quantitative RT-PCR from mice liver. TPO expression was higher in the group that received quinine and guava extract compared to quinine only, although this difference was not statistically significant ( $p>0.05)$.

Data were presented from 5 independent mice in each group.

Q: quinine, GE: guava extract, DW: distilled water, NS: not significant

\section{DISCUSSION}

The 14-d quinine induction with a dosage of $2.8 \mathrm{mg} / 20 \mathrm{~g}$ body weight reduced the platelet count in our mice model. Quinine is known to cause an immune system-mediated toxic thrombocytopenia. The antibodies responsible for quinine-induced thrombocytopenia are immuneglobulins that recognize the fibrinogen receptor glycoprotein (GP) Ib/IX or GPIIb/IIIa. The platelet antigens (GPIb/IX and GPIIb/IIIa) are also expressed on MKs during differentiation. Therefore, it is likely that quinine may also bind to cell surface GPs on MKs and inhibits megakaryopoiesis, resulting in a decrease in platelet count [17]

Platelet generation is a two-stage process consisting of megakaryopoiesis and thrombopoiesis [4]. Megakaryopoiesis is a complex process that involves differentiation of hematopoietic stem cells (HSCs) to the megakaryocyte (MK), the proliferation of the progenitors, and MK maturation [18]. SCF is a cytokine critical during differentiation process of hematopoietic stem cells (HSCs) into mature blood cells. SCF promotes survival, proliferation and mobilization of hematopoietic stem cells and their progenitors. Interacting with other cytokines, such as interleukin (IL)-1, IL-3, IL-6, and GM-CSF, SCF promotes differentiation and maturation of common myeloid progenitor (CMP) into colony-forming-unit megakaryocyte (CFU-MK), the primitive and mature MKs progenitors [19]. Platelet production occurs when the cytoplasm of MKs extends and forms a prothrombocyte [18]. TPO is the major cytokine on this process. Previous evidence showed that TPO act in conjunction with another cytokine, such as IL-3, IL-6, and IL-11, however, these cytokines are not essential for platelet production [13]

Our study showed that after the $14^{\text {th }}$ day of treatment, mice in the group that received quinine and guava extracts demonstrated an increase in platelet count compared to mice in the quinine only group. We postulate that this difference is due toguava extract administration. Guava is a medicinal plant that contains vitamin $C$ vitamin A, iron, calcium and phosphor, manganese, saponin combined with an oleanolic acid, morin-3-0- $\alpha$-L-lyxopyranoside and morin-3-0- $\alpha$-L-arabopyranoside and flavonoids, guaijavarin, and quercetin [5]. The previous study showed that quercetinin guava increased the number of Mks and thrombocytes [20].

Likewise, one other study showed that the combination of $2.8 \mathrm{mg} / 20$ g body weight/d quinine orally for 14 dand $0.785 \mathrm{mg} / 20 \mathrm{~g}$ body weight/d guava extract for 5 dresulted inan increase in the average number of MKs compared to the group were received quinine only [9]. Quercetin has been known to increase SCF mRNA expression in bone marrow stromal cells [10]. In addition to hematopoiesis, SCF is also involved in hepatocyte proliferation. The previous study showed that SCF mRNA expression in mice liver increased after $6 \mathrm{~h}$ of acetaminophen administration and SCF increased hepatocyte proliferation after acetaminophen overdose [21].

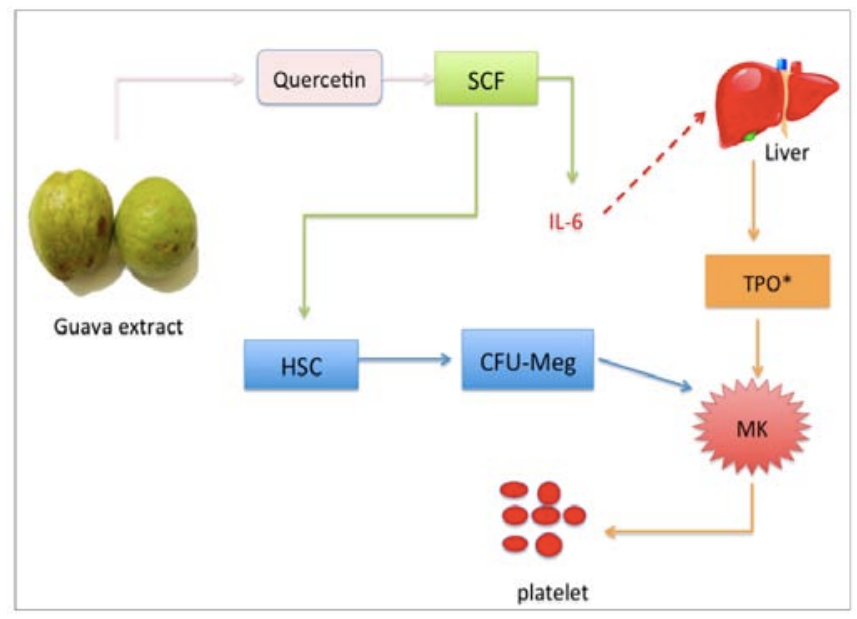

Fig. 5: Proposed mechanism of platelet production by guava extract, administration of guava extract rich in quercetin increase level of SCF mRNA which can stimulate IL-6 to induce TPO secretion by liver resulting an increase the platelets number, *to be further confirm 
Although megakaryopoiesis and thrombopoiesis occur in bone marrow, TPO is synthesized by hepatocytes in the liver [13], hence, the use of liver to determine SCF and TPO mRNA expression in our study. Previously, it was assumed that SCF stimulates platelet production by increasing IL-6 mast cell secretion which in turn induces liver to secrete thrombopoietin, a major thrombopoiesis cytokine (fig. 5).

Our findings showed that SCF mRNA expression increased only in the groups that received quinine and guava extract and had no effect on mice that received distilled water and guava extract. This can be interpreted that guava extract stimulates SCF mRNA expression and platelet number only in thrombocytopenia models. However, enhancement of SCF mRNA expression was not followed by an increase of TPO analytically, but based on a Livak method that TPO mRNA expression also increased in the group treated with quinine and guava extract. This is probably caused by of the small sample size or the presence of other molecular mechanisms that may increase platelet production by guava extracts, such as IL-3, IL-6, and IL-1. As a follow-up, we will examine several molecule candidates to pinpoint this mechanism, in addition to increasing the sample size.

\section{CONCLUSION}

Guava extract can increase platelet number of thrombocytopenic mice model through enhancement of stem cell factor expression as one of the molecular pathway. Furthermore, the extent of study is necessary to be elucidated more about the benefit of guava extract in a thrombocytopenic patient such as Dengue fever.

\section{ACKNOWLEDGEMENT}

The authors thank Nurul for technical assistance and Dr. Panji Fortuna for helpful discussions. The project was supported by the Internal Grant of Universitas Padjadjaran (855/UN6.3.1/PL/2017).

\section{AUTHOR CONTRIBUTIONS}

NUR ATIK: Designed and conducted the experiments, prepared the manuscript.

SUSAN TARAWIFA: Conducted the experiments and prepared the manuscript.

ERDA AVRIYANTI: Prepared the manuscript.

ANDRI REZA RAHMADI: Designed the experiments.

DANY HILMANTO: Designed the experiments and prepared the manuscript.

\section{CONFLICT OF INTERESTS}

\section{Declared none}

\section{REFERENCES}

1. Machlus KR, Italiano JE. The incredible journey: from megakaryocyte development to platelet formation. J Cell Biol 2013;201:785-96.

2. Guyton AC, Hall JE. Textbook of Medical Physiology. $13^{\text {th }}$ ed. Philadelphia; Elsevier; 2016.
3. Eto K, Kunishima S. Linkage between the mechanisms of thrombocytopenia and thrombopoiesis. Blood 2016;127:1234-41.

4. Drayer AL, Boer AK, Los L. Stem cell factor synergistically enhances thrombopoietin - induced STAT5 signaling in megakaryocyte progenitors through JAK2 and src kinase. Stem Cells 2005;23:240-51.

5. Shruthi DS, Roshan A, Timilsina SS, Sunita S. A review on the medicinal plant Psidiumguajava Linn. (Myrtaceae). Drug Delivery Ther 2013;3:162-8.

6. Rabbaniyah F. The effect of leaf extract guava (Psidium guajava Linn.) against increased platelets in patients with dengue hemorrhagic fever. Majority 2015;4:91-6.

7. Kummee P, Borisutpeth M, Chanlun S, Kanbutra P, Chanlun A. Efficacy of guava leaf extract as alternative pre-milking teat dipping in reducing teat-end bacterial load of milking dairy cows. Int J Pharm Pharm Sci 2015;7:434-8.

8. Prasetio JN. Potential red guava juice in a patient with dengue hemorrhagic fever. Majority 2015;4:25-9.

9. Atik N, Durry M, Tarawifa S, Darmadji HP. The effect of guava extract administration on megakaryocytes amount in mice femur. Indonesian J Clin Pharm 2017;6:116-22.

10. Shin S, Kim T, Youm S, Kim Y, Ahn B. Quercetin promotes the differentiation of hematopoietic stem cells or early progenitor cells into erythroid lineage in mice. J Med Plant 2011;21:76-83.

11. MacNeil AJ, Junkins RD, Wu Z, Lin TJ. Stem cell factor induces AP-1-dependent mast cell IL-6 production via MAPK kinase 3 activity. J Leuk Biol 2014;95:903-15.

12. Kaser A, Brandacher G, Steurer W. Interleukin-6 stimulates thrombopoiesis through thrombopoietin: role in inflammatory thrombocytosis. Blood 2001;98:2720-5.

13. Kaushansky $\mathrm{K}$. The molecular mechanisms that control thrombopoiesis. J Clin Invest 2005;15:3348-54.

14. Ghai CL. Textbook of practical physiology. $2^{\text {nd }}$ ed. New Delhi; Jaypee Brothers Medical Publisher; 2013.

15. Stoffel R, Wiestner A, Skoda RC. Thrombopoietin in thrombocytopenic mice: evidence against regulation at the mRNA level and for a direct regulatory role of platelets. Blood 1996;87:567-73.

16. Wang $\mathrm{CH}$, Verma S, Hsieh IC. Stem cell factor attenuates vascular smooth muscle apoptosis and increases intimal hyperplasia after vascular injury. Arterioscler Thromb Vasc Biol 2007; 27:540-7.

17. Perdomo J, Yan F, Ahmadi Z, Jiang XM, Stocker R, Chong BH. Quinine-induced thrombocytopenia: drug-dependent Gpib/IX antibodies inhibit megakaryocyte and proplatelet production in vitro. Blood 2011;117:5975-86

18. Guo T, Wang X, Qu Y. Megakaryopoiesis and platelet production: insight into hematopoietic stem cell proliferation and differentiation. Stem Cell Invest 2015;2:3.

19. Patel S, Hartwig JH, Italiano Jr JE. The biogenesis of platelets from megakaryocyte proplatelets. J Clin Invest 2015;115:3348-54.

20. Soegijanto S, Tumbelaka AR, Anggraini RR, Sary DD. Multicenter clinical trial of guava leaf extract in dengue hemorrhagic fever patients. Medicinus 2010;23:5-10.

21. $\mathrm{Hu} \mathrm{B}$, Colleti LM. Stem cell factor and c-kit are involved in hepatic recovery after acetaminophen-induced liver injury in mice. Am J Physiol Gastrointest Liver Physiol 2008;295:45-53. 